\title{
Histoire de la maréchaussée et de la gendarmerie. Guide de recherche
}

\section{Bernard Gainot}

\section{(2) OpenEdition \\ 9 Journals}

Édition électronique

URL : https://journals.openedition.org/ahrf/1961

DOI : 10.4000/ahrf.1961

ISSN : 1952-403X

\section{Éditeur :}

Armand Colin, Société des études robespierristes

\section{Édition imprimée}

Date de publication : 1 décembre 2005

Pagination : 253-255

ISSN : 0003-4436

\section{Référence électronique}

Bernard Gainot, «Histoire de la maréchaussée et de la gendarmerie. Guide de recherche », Annales historiques de la Révolution française [En ligne], 342 I octobre-décembre 2005, mis en ligne le 05 avril 2006, consulté le 22 avril 2022. URL : http://journals.openedition.org/ahrf/1961 ; DOI : https://doi.org/ 10.4000/ahrf.1961

Ce document a été généré automatiquement le 22 avril 2022.

Tous droits réservés 


\title{
Histoire de la maréchaussée et de la gendarmerie. Guide de recherche
}

\author{
Bernard Gainot
}

\section{RÉFÉRENCE}

Jean-Noël LUC (dir.), Histoire de la maréchaussée et de la gendarmerie. Guide de recherche, Maison Alfort, Service Historique de la Gendarmerie Nationale, 2005, 1105 p., ISBN :

2-11-095130-3, $33 €$

1 C'est un volume tout à fait impressionnant que nous livre présentement le Service Historique de la Gendarmerie Nationale (SHGN); certes par sa taille (plus de mille pages), mais surtout par sa qualité. Fruit du travail, depuis 1998, d'une équipe de trente-trois auteurs qui associe universitaires, chargés de recherches appartenant pour la plupart à l'institution, doctorants et docteurs bien au fait du sujet, le produit fini, dont Jean-Noël Luc fut le concepteur, puis le maître d'œuvre, le chef d'escadron Édouard Ebel le coordinateur sur le plan scientifique, et le chef d'escadron Antoine Boulant le coordonnateur éditorial, déborde très largement le qualificatif de "guide " auquel semble le réduire le sous-titre. C'est tout à la fois un outil de travail indispensable aux chercheurs intéressés par les dimensions juridiques, sociales, culturelles, politiques, du maintien de l'ordre, un état actuel de la recherche dans ces domaines, et un essai historiographique, véritable plaidoyer pour initier à une étude novatrice, et scientifique, débarrassée de toutes les velléités hagiographiques, auxquelles on cherche encore bien souvent, non sans un mépris manifeste, à la confiner.

2 Qu'on en juge. L'ouvrage est divisé en six parties. La première partie (presque le tiers $\mathrm{du}$ volume) présente "l'institution"; on insistera particulièrement sur les dix-huit organigrammes, qui font le point sur l'organisation de la gendarmerie aux momentsclefs de son évolution depuis 1791, les repères chronologiques depuis la guerre de Cent Ans jusqu'au temps présent, les grands textes qui ont fixé organisation et missions 
depuis 1791. Très significative également est la sous-partie concernant les uniformes et la symbolique (drapeaux, insignes, couleurs, devises), qui insère un champ très visité par l'histoire militaire traditionnelle dans une perspective renouvelée d'histoire culturelle.

La deuxième partie porte sur la «bibliographie»; une centaine de pages d'un inventaire exhaustif des ouvrages ayant la maréchaussée et la gendarmerie pour objet, mais également des divers travaux universitaires aboutis, et, ce qui est encore plus important, des travaux en cours sur la question. La troisième partie (170 pages) est un inventaire des fonds d'archives; Laurent Veyssière, conservateur aux Archives nationales, retrace dans un article l'historique de ces précieux dépôts qui reviennent de loin («Les archives de la gendarmerie, de la destruction à la sauvegarde »), illustrant parfaitement la démarche méthodologique, qui veut que chaque recherche innovante construise ses propres sources. Ainsi, outre les fonds bien identifiés aux Archives nationales, au Service historique de l'armée de terre (désormais restucturé, avec le SHGN, et d'autres services spécialisés dont il est question dans cette partie, dans un organisme intégré nommé "Service Historique de la Défense»), aux Archives départementales, on retiendra tout particulièrement les fonds de la Bibliothèque Historique de la Ville de Paris (très riches pour la période révolutionnaire), ou ceux de la Bibliothèque de l'Arsenal (pour les arts du spectacle). La sous-partie intitulée "Sources des études prosopographiques " suggère de très utiles pistes de recherches sur un chantier particulièrement novateur.

4 La quatrième partie, d'ampleur équivalente (165 pages), rassemble les «autres sources "; on passera sur le caractère un peu fourre-tout de cette rubrique (regroupement des sources imprimées, des sources iconographiques et des sources orales), pour insister plutôt, là aussi, sur les suggestions innovantes : le répertoire des nombreux mémoires de gendarmes (ou ayant trait à des gendarmes), des romans, des pièces de théatre, y compris des spectacles de marionnettes (le merveilleux corpus des Guignol et Polichinelle), de la filmographie (219 apparitions de gendarmes sur grand écran de 1899 à 2003 !), des poèmes et chansons (Gustave Nadaud, le concepteur du célèbre Pandore en 1852, mais Brassens et Bourvil sont également en bonne place).

inquième partie est une exploration des «lieux de mémoire » de l'institution (35 pages) ; musées, salles d'honneur, monuments, stèles et plaques commémoratives, qui fixent les discours que la gendarmerie élabore sur elle-même, et présentent les matériaux pour l'élaboration des représentations qu'elle donne d'elle-même. Enfin, une sixième partie (une centaine de pages) est un lexique des réalités institutionnelles propres à la maréchaussée et à la gendarmerie, un « dictionnaire historique ».

6 Chacune de ces parties est un bilan en soi, état des travaux et perspectives de recherches. Pour l'état des travaux, le guide illustre brillamment les efforts conjoints du séminaire que Jean-Noël Luc anime depuis 1999 à Paris IV, et du SHGN, créé en 1995. Nous en avons d'autres témoignages; le grand colloque de la Sorbonne en 2000 (dont les actes ont été publiés en 2002 sous le titre Gendarmerie, État et société au XIXe siècle par les Publications de la Sorbonne, et qui a fait l'objet d'un compte rendu dans un précédent numéro de la revue), les très nombreuses publications du SHGN de MaisonsAlfort, qui attestent de son dynamisme, et dont nous rendons compte également dans notre revue, pour celles du moins qui se rapportent à la période révolutionnaire et impériale, comme la réédition de l'Histoire de la maréchaussée et de la gendarmerie du général Louis Larrieu. 
7 En introduction, J.N. Luc présente un véritable manifeste historiographique et problématique intitulé "Pour une histoire de la gendarmerie»; tout travail scientifique sur le maintien de l'ordre, toute recherche sur la maréchaussée et la gendarmerie doivent désormais s'y référer. Après un bref aperçu des origines de l'»arme» (l'auteur s'explique sur les ambiguïtés dans l'usage de ce terme), il s'interroge sur les raisons pour lesquelles « Clio a [...] longtemps boudé les gendarmes » (p. 20) ; sujet «trop militaire pour les uns [...] pas assez pour les autres» (p. 23). Puis il s'attache à mesurer les conditions et l'ampleur de la réconciliation entre clio et Pandore au cours de la dernière décennie. Au total, ce sont 115 travaux qui sont répartis par champs d'études (p. 28). Enfin, J.N. Luc propose des pistes de recherches, dans une perspective interdisciplinaire, dans plusieurs domaines sélectionnés : «la force publique, l'instrument de l'intégration nationale, l'institution militaire, la communauté socioprofessionnelle et les représentations dont elle est l'objet » (p. 29). L'enjeu de la vaste problématique esquissée est de partir du lieu institutionnel, la brigade, la caserne, l'unité combattante, pour mieux admettre «l'historicité de l'organisation, des pratiques et des images de la gendarmerie. Cher au discours officiel, le principe de continuité ne doit pas faire oublier l'ampleur des mutations, politiques, économiques, sociales, démographiques, culturelles, auxquelles cette institution a été confrontée » (p. 34).

8 Dans ce bilan global, l'auteur a recensé seize travaux universitaires portant sur la période révolutionnaire et impériale, soit 13\% environ de la période 1789-1914. Sur ces seize travaux, quatre ont été réalisés dans le cadre de l'Institut d'histoire de la Révolution française de Paris I, soit un nombre équivalent à ceux qui ont été réalisés dans le cadre du séminaire de Paris IV, ce qui méritait d'être relevé. Pour autant, ce domaine de recherches reste marginal pour la décennie révolutionnaire : c'est la période impériale qui se taille la part du lion (sept travaux), puis les études qui prennent la grande loi organique de 1798 comme point de départ (six travaux). Il reste donc trois études consacrées à cette période 1791 - 1798, certes confuse, certes difficile d'accès en raison du caractère lacunaire et dispersé des sources, mais riche d'expérimentations novatrices (la division des pouvoirs, le renseignement, la formation des unités combattantes, le choix des cadres). Elle n'est somme toute guère mieux lotie que la Monarchie de Juillet. Le guide est précisément un bon tremplin pour susciter les vocations en ce domaine, quitte à compléter, ou rectifier certains points de détail. Il semble, notamment, que dans la chronologie, une place doit être faite à la grande loi sur la police intérieure des communes, du 10 vendémiaire an IV ( 2 octobre 1795), car elle eut une incidence fondamentale pour les missions de la gendarmerie en zone rurale, au moins pendant toute la première moitié du XIXe siècle. Quant aux « colonnes mobiles », elles ne furent pas créées en 1798 " pour faire appliquer la loi Jourdan sur la conscription ", comme il est dit p. 931, mais par l'arrêté du Directoire du 17 floréal an IV (6 mai 1796) (Bulletin des lois, XLIX, $\mathrm{n}^{\circ}$ 410) pour réprimer le banditisme et la subversion intérieure. Ces quelques remarques pointillistes n'altèrent en aucune façon le grand intérêt scientifique de ce travail collectif. Nous partageons entièrement l'appréciation du général Quentel, chef du SHGN depuis 2003 : "Cet ouvrage n'a aujourd'hui pas d'équivalent dans les armées, ni dans les autres corps de l'État ». 